\title{
Mixed Perturbative Expansion: the Validity of a Model for the Cascading
}

\author{
Hervé LEBLOND \\ Laboratoire POMA, UMR CNRS 6136, Université d'Angers, \\ $2 B^{d}$ Lavoisier 49045 ANGERS Cedex 01, France \\ E-mail: herve.leblond@univ-angers.fr
}

Received May 31, 2001; Revised September 17, 2001; Accepted December 11, 2001

\begin{abstract}
A new type of perturbative expansion is built in order to give a rigorous derivation and to clarify the range of validity of some commonly used model equations. This model describes the evolution of the modulation of two short and localized pulses, fundamental and second harmonic, propagating together in a bulk uniaxial crystal with non-vanishing second order susceptibility $\chi^{(2)}$, and interacting through the nonlinear effect known as "cascading" in nonlinear optics. The perturbative method mixes a multi-scale expansion with a power series expansion of the susceptibility, and must be carefully adapted to the physical situation. It allows the determination of the physical conditions under which the model is valid: the order of magnitude of the walk-off, phase-mismatch, and anisotropy must have determined values.
\end{abstract}

\section{Introduction}

\section{$1.1 \quad$ A methodological problem}

Many scientists, in recent times, came to the idea that physics is intrinsically nonlinear and that most of the linear theories have been introduced because they were mathematically tractable, contrarily to the nonlinear problems. Nowadays, the theoreticians of nonlinear physics almost always try to keep the largest part of the ideas involved by the linear theories of the physical problems considered. Both mathematical tools and physical concepts are conserved. The nonlinear theory appears then mainly as a perturbative approach in which the linear theory is the zero order. As an essential example, the Fourier theory and the plane monochromatic wave concept are the main tools in the linear theory of wave propagation. The theory of nonlinear wave propagation considers some wave packet, that is in fact a monochromatic plane wave (i.e. the linear wave) modulated by some slowly varying envelope (which is thus some first correction to the linear theory). This theory involves thus at least two space scales: that of the wavelength and that of the pulse length, which are treated separately by the mathematical model. The natural frame for the derivation of the nonlinear models is thus the so-called multiscale expansion method. From the mathematical viewpoint, formal computations in this frame can be performed 
in a perfectly rigorous way. Convergence proofs have also been given [5]. They enhance and clarify the validity of the formal computations.

A multiscale expansion, as well as any asymptotic expansion, has a structural drawback. It involves some small parameter $\varepsilon$, that is infinitely small in the mathematical theory, while it takes a small but finite value in the physical applications. Consider then some physical quantity, small in relation to the physical value of $\varepsilon$, but independent of it. Because it is finite while $\varepsilon$ is not, the mathematical theory considers it de facto as if it were large in relation to $\varepsilon$. This may sometimes introduce erroneous results, in so far that some further approximation is not taken into account. To introduce, beside the slowly varying envelope approximation and mixed to it, a further approximation of another type yields great technical difficulties. Such an approach mixing a multiscale expansion to an approximation of another type has already been done for the treatment of damping for soliton propagation, in particular for electromagnetic waves in ferromagnets [15, 10]. The damping parameter is identified to some power of the perturbative parameter $\varepsilon$, and all involved quantities are expanded in a power series of the damping constant.

In other situations, the ansatz must be constructed through a careful analysis of the physical situation. We study in this paper a situation in which such a mixed expansion is needed. It is presented from a methodological point of view, and should be of interest for the readers who intend to apply the same kind of perturbative approach to other physical situations. The proper physical consequences of the result will be published elsewhere [13].

\subsection{Solitons through cascading}

The physical situation we consider is the propagation of optical solitons using the socalled cascading phenomenon, viz. second-harmonic generation and back-conversion to the fundamental, that arises in a dielectric medium with a non-vanishing second-order nonlinear susceptibility $\chi^{(2)}$. Several theoretical results have been published in order to show that this phenomenon could be responsible for the stabilization of a short pulse and for solitonic behavior [1, 9, 14, 16]. These theoretical works study by analytic and numerical methods some partial differential system, derived in the frame of the slowly varying envelope approximation, and analogous in its form to the nonlinear Schrödinger (NLS) equation, at least for the linear part. The nonlinear part of this system gives account for the interaction between the fundamental and the second harmonic. Some applications of this model have been found in good agreement with experimental results. However, the way it is derived in the literature is quite phenomenological. It appears thus to be worth giving a rigorous formal derivation of that model, and to clarify its range of validity.

Two waves propagating together are considered here, and their interaction is expected to stabilize the pulse. Therefore two conditions must be satisfied, at least in an approximate way. First the interaction must be resonant, thus the waves must be phase-matched. If not the interaction will not be strong enough to yield the expected effect. Second the two waves must propagate together during a long enough time. Thus their group velocities must be equal, or at least very close together. We determine precisely the order of magnitude of the allowed small deviation from phase-matching and small walk-off. We restrict for technical reasons our attention to crystals having some particular symmetry. However, the required phase and group velocity matching conditions cannot be realized in any crystal of this type: the linear dispersion relation of the material must satisfy several 
conditions. The order of magnitude of some physical quantities must be comparable to powers of the perturbative parameter characteristic of the multiscale expansion.

\section{Analysis of the physical problem}

\subsection{The equations for a uniaxial optical crystal}

We consider a bulk crystal with a non-zero second order susceptibility $\left(\chi^{(2)}\right)$ tensor, optically uniaxial from the linear point of view and perfectly transparent. These hypotheses are satisfied by potassium dihydrogen phosphate (KDP) and analogous materials or by lithium niobate, as examples. Wave propagation in such a medium is described by the Maxwell equations that reduce to

$$
\vec{\nabla} \wedge(\vec{\nabla} \wedge \vec{E})=-\frac{1}{c^{2}} \partial_{t}^{2}(\vec{E}+\vec{D})
$$

where $c$ is the speed of light in vacuum, and

$$
\begin{gathered}
\vec{D}=\chi^{(1)} * \vec{E}+\chi^{(2)} * \vec{E} \vec{E} \\
=\int_{-\infty}^{t} \chi^{(1)}\left(t-t^{\prime}\right) \vec{E}\left(t^{\prime}\right) d t^{\prime}+\int_{-\infty}^{t} \int_{-\infty}^{t^{\prime}} \chi^{(2)}\left(t-t^{\prime}, t-t^{\prime \prime}\right): \vec{E}\left(t^{\prime}\right) \vec{E}\left(t^{\prime \prime}\right) d t^{\prime \prime} d t^{\prime}
\end{gathered}
$$

$\chi^{(1)}$ and $\chi^{(2)}$ are respectively the linear and the quadratic susceptibility tensors 4 . We denote by $\hat{\chi}^{(1)}(\omega)$ the Fourier transform of $\chi^{(1)}(t)$ so that $\chi^{(1)}(t)=\int_{-\infty}^{+\infty} \hat{\chi}^{(1)}(\omega) e^{i \omega t} d \omega$. Then, for a uniaxial crystal, the coordinate frame being chosen so that the optical axis is the $z$-axis, the linear susceptibility tensor satisfies

$$
1+\hat{\chi}^{(1)}=\left(\begin{array}{ccc}
n_{o}^{2} & 0 & 0 \\
0 & n_{o}^{2} & 0 \\
0 & 0 & n_{e}^{2}
\end{array}\right) .
$$

$n_{o}(\omega)$ and $n_{e}(\omega)$ are called respectively the ordinary and extraordinary indices [2]. A wave propagates along the optical axis with the velocity $c / n_{o}$. If the propagation direction makes some nonzero angle $\theta$ with this axis, two waves can propagate, with different speeds and polarizations. They are referred to as ordinary and extraordinary waves. The characteristic feature of the extraordinary wave is that its group velocity is not parallel to its phase velocity, which never happens in isotropic media. If $\theta=\pi / 2$, the wave polarized perpendicular to the optical axis is the ordinary one, the wave polarized along the axis is extraordinary, and propagates with the velocity $c / n_{e}$.

\subsection{The phase-matching angle}

No wave interaction can occur far from the phase resonance, or phase-matching. Therefore we must work close to it. We denote by $v_{\varphi}(\omega)$ and $v_{\varphi}(2 \omega)$ the phase velocities of the fundamental and second harmonic respectively. The phase-matching condition obviously is

$$
v_{\varphi}(\omega)=v_{\varphi}(2 \omega)
$$


It is well-known 177 that this phase-matching condition can be realized by some adequate choice of the value of the angle $\theta$ between the optical axis (z-axis) and the propagation direction. Several situations arise, depending on the relative magnitude of the indices in the considered material and on the polarization of both waves. We consider only one of these cases, of major importance for applications. For KDP and many other materials, the inequalities $n_{o}(2 \omega)>n_{o}(\omega)$ and $n_{o}(\omega)>n_{e}(\omega)$ are satisfied. It is easily shown that in this case the phase-matching angle exists when the fundamental wave is ordinary and the second-harmonic extraordinary, but does not exist for other polarizations, except if both waves are extraordinary. In the latter situation, the experimental values of $n_{e}$ and $n_{o}$ do not yield a real phase-matching angle value. We restrict thus the study to the particular case where the fundamental wave is ordinary and the second-harmonic extraordinary, which is the only physical situation in which the phase-matching angle exists for the materials under consideration. The phase-matching condition is then [17]

$$
\sin ^{2} \theta=\frac{n_{o}^{-2}(\omega)-n_{o}^{-2}(2 \omega)}{n_{e}^{-2}(2 \omega)-n_{o}^{-2}(2 \omega)}
$$

The first condition to be satisfied by our ansatz is that the angle $\theta$ between the propagation direction and the $z$-axis must satisfy the phase-matching condition (2.5), at least in an approximate way.

\subsection{Transverse velocity}

The dispersion relation $\omega(\vec{k})$ follows from $(2.3)$, the value of the group velocity is then computed according to $\vec{v}=\partial \omega / \partial \vec{k}$. While the group velocity of the ordinary wave is parallel to the phase velocity, that of the extraordinary wave has a transverse component [17]. It is

$$
v_{e, x}=\frac{c^{2} k}{2 \Lambda}\left(\frac{1}{n_{e}^{2}}-\frac{1}{n_{o}^{2}}\right) \sin 2 \theta
$$

with

$$
\Lambda=\omega^{2}+c^{2} k^{2}\left(\frac{\cos ^{2} \theta n_{o}^{\prime}}{n_{o}^{3}}+\frac{\sin ^{2} \theta n_{e}^{\prime}}{n_{e}^{3}}\right)
$$

( $k$ is the wave vector and $\omega$ the pulsation of the fundamental, $n_{o}^{\prime}=d n_{o} / d \omega$ ). Because we expect that the walk-off is small, $v_{e, x}$ must be close to zero. This can be achieved in two ways: either by setting $n_{e}$ close to $n_{o}$, or when $\sin 2 \theta$ is small. Try first to satisfy the former condition: anisotropy must be weak. However, anisotropy is needed for the phase-matching that must be realized at least in an approximate way. The difference $\left(n_{e}-n_{o}\right)$ appears indeed in the denominator of (2.5), and therefore it cannot be zero. The anisotropy, or the difference $\left(n_{e}-n_{o}\right)$, must be small but not too small. This is the second condition imposed to our ansatz. It can be satisfied in many real materials.

It is likely also necessary that $\sin 2 \theta$ is small. We note in (2.5) that, when $n_{e}(2 \omega)=$ $n_{o}(\omega)$, then $\sin ^{2} \theta=1$. In this case $\theta=\pi / 2$ and $\sin 2 \theta=0$. We deduce this way a third condition to be satisfied by the ansatz: $n_{e}(2 \omega)$ must be close to $n_{o}(\omega)$. Thus the achievement of phase-matching and reduction of walk-off necessitate that $n_{o}(2 \omega)$ and $n_{e}(\omega)$ are very close together, and that anisotropy is weak. The ansatz used below is built in order to satisfy these conditions. It is seen from published experimental data [6] that they 
are realized in many real materials which are in fact the most commonly used for practical applications of the second harmonic generation. Further it was very early recognized that the arising of the transverse group velocity or walk-off is the main limitation to an efficient second harmonic generation at phase-matching angle [3], and the fact that the condition " $n_{e}(2 \omega)$ close to $n_{o}(\omega)$ " ensures the reduction of the walk-off in this situation follows straightforwardly from the properties of light propagation in a birefringent medium.

\section{An adequate ansatz}

\subsection{The scaling parameter}

As usual in the frame of multiscale analysis, we introduce some small parameter $\varepsilon$. It characterizes the pulse size in the following way: we call $L$ the common order of magnitude of the pulse length and width, and $\lambda$ the wavelength. Then the perturbative parameter is defined as

$$
\varepsilon=\frac{\lambda}{L}
$$

The electric field $\vec{E}$ is expanded both in a power series of some small parameter $\varepsilon$, and in a Fourier series of some fundamental phase $\phi$ as

$$
\vec{E}=\sum_{l \geq l_{0}, p \in \mathbb{Z}} \varepsilon^{l} \vec{E}_{l}^{p} e^{i p \phi}
$$

$l_{0}$ fixes the order of magnitude of the main term. It is discussed below. The amplitudes $\vec{E}_{l}^{p}$ are functions of slow variables that are defined below. The main slow variables characterize the pulse shape and its propagation at the group velocity. According to (3.1), they are variables of order $\varepsilon$, defined by

$$
\xi=\varepsilon x, \quad \eta=\varepsilon y, \quad \zeta=\varepsilon z, \quad \tau=\varepsilon t .
$$

We seek for the evolution of the pulse shape through propagation distances long in relation to the pulse length $L$, say $L / \varepsilon$, as in the standard nonlinear Schrödinger model. On these grounds and because the propagation direction is close to the $x$-axis (see below), the slow variable giving account for the evolution can be chosen as

$$
\xi_{2}=\varepsilon^{2} x .
$$

\subsection{The carrier}

The phase $\phi$ determines the carrier monochromatic plane wave. It thus determines the propagation direction. Recall that, because phase-matching must be at least approximately realized, the angle $\theta$ between the $z$-axis and the propagation direction must be close to the value given in (2.5). On the other hand, it has been shown in subsection 2.3 that the latter value must be close to $\pi / 2$. We write it as

$$
\theta=\frac{\pi}{2}-\varepsilon^{a} \gamma,
$$


where $\gamma$ is a free parameter at this point, and the exponent $a$ is positive and is determined below. The phase $\phi$ is

$$
\phi=\vec{k} \cdot \vec{x}-\omega t,
$$

with $\vec{k}=k(\sin \theta, 0, \cos \theta)$. Inserting the definition (3.5) of $\theta$ into the expression (3.6) of $\phi$, we would get

$$
\phi=k x+k \gamma \varepsilon^{a} z-\omega t+O\left(\varepsilon^{2 a}\right) .
$$

A dependency with regard to some slow variable $\varepsilon^{a} z$ appears $(a>0)$. For $a \geq 1$, this dependency can be incorporated in the amplitude, that depends on $\zeta=\varepsilon z$. Thus only values of $a$ less than 1 are interesting. On these grounds, and taking into account the fact that the perturbative expansion will involve only integer powers of $\varepsilon^{a}$, it is seen that the correct choice for $a$ is $a=1 / 2$. Further, due to the anisotropy, the wave vector norm $k$ depends on the angle $\theta$. Thus, according to (3.5), $k$ depends on $\varepsilon$. As $\varepsilon$ tends to $0, k$ must be expanded in a power series of $\sqrt{\varepsilon}$. A term involving $\sqrt{\varepsilon} x$ appears then in (3.7). The terms of order $\varepsilon^{1}$ and higher are incorporated in the amplitude. The complete phase $\phi$ can thus be written as

$$
\phi=k(1+\alpha \sqrt{\varepsilon}) x+\sqrt{\varepsilon} k \gamma z-\omega t .
$$

In the same way as $k$, all quantities such as group velocities or polarization vectors, that depend on the angle $\theta$, are expanded in a power series of $\sqrt{\varepsilon}$. Therefore we must introduce an additional slow variable of half-integer order,

$$
\xi_{1}=\varepsilon^{\frac{3}{2}} x .
$$

The complete set of slow variables on which the amplitudes $\vec{E}_{l}^{p}$ depend is thus $\left(\xi, \eta, \zeta, \tau, \xi_{1}, \xi_{2}\right)$. They are defined by (3.3), (3.4), and (3.9).

Leaving aside the half integer powers of $\varepsilon$, this is mainly the "classical" multiscale expansion that leads ordinary to the nonlinear Schrödinger-type (NLS-type) models ([0], p. $495 \mathrm{sq}$.) describing long-distance propagation along the $x$-axis. Recall indeed that it is not necessary to introduce a priori the statement that the field is at first order a function of $(x-V t)$. Else, the linear transport equation, that is the equation giving account for the modulation propagation at group velocity, is obtained as a solvability condition of the perturbative scheme. The main originality of the expansion (3.2)-(3.9) is the introduction of an intermediate scale $x \sqrt{\varepsilon}, z \sqrt{\varepsilon}$ in the expression of the phase $\phi$. The expansion therefore involves not only 3 scales for the longitudinal space variable, as in the usual NLS-type expansions, but 5 different scales (including $\xi_{1}$ ). The term $\sqrt{\varepsilon} k \gamma z$ in expression (3.8) for $\phi$ represents a deviation for an angle $\sqrt{\varepsilon} \gamma$ from the propagation along the $x$-axis.

\subsection{Expansion of the susceptibility}

Here comes the difficult point which is the introduction of a second approximation beside the multiscale expansion. It has been seen that the components of the linear susceptibility tensor must satisfy several hypotheses that allow certain approximations to be made. The problem is that the only consistent way of describing mathematically these hypotheses requires the $\chi^{(1)}$ tensor to be written as a function of the perturbative parameter $\varepsilon$. However, $\varepsilon$ represents the pulse size, and $\chi^{(1)}$ does not depend on it from the physical 
point of view. Nevertheless, we assume that the linear susceptibility tensor $\chi^{(1)}$ can be formally expanded in a power series of $\sqrt{\varepsilon}$ in the following way:

$$
1+\hat{\chi}^{(1)}=n^{2}+\sqrt{\varepsilon} \hat{\chi}_{\frac{1}{2}}^{(1)}+\varepsilon \hat{\chi}_{1}^{(1)}+\varepsilon^{\frac{3}{2}} \hat{\chi}_{\frac{3}{2}}^{(1)}+\cdots
$$

$n^{2}$ is an isotropic index, and the fact that $\lim _{\varepsilon \rightarrow 0}\left(1+\hat{\chi}^{(1)}\right)=n^{2}$ gives a partial account for the second condition of $\S 2.3$, the weak anisotropy hypothesis. A particular definition of the coefficients $\hat{\chi}_{j}^{(1)}$ can be given. It is of no interest for our purpose, and is left for further publication 13.

\subsection{The amplitude}

An important difference between the ansatz (3.2) and the standard one for NLS is the order of magnitude of the leading term, that is the value of $l_{0}$ in (3.2). It is determined as follows. The model that we intend to derive and to justify is used in [16, 14, 9]. It has the following form:

$$
\begin{gathered}
i \partial_{\xi_{2}} \varphi+D_{\vec{\xi}}^{2} \varphi=A_{1} \psi \varphi^{*}, \\
i \partial_{\xi_{2}} \psi+D_{\vec{\xi}}^{\prime 2} \psi=A_{2} \varphi^{2}
\end{gathered}
$$

where $D_{\vec{\xi}}^{2}$ and $D_{\vec{\xi}}^{\prime 2}$ are second-order partial differential operators relative to the slow variables $(\tau-\xi / V), \eta, \zeta$ that describe the shape of the pulse. $A_{1}, A_{2}$ are some constants. $\varphi$ and $\psi$ are the amplitudes of the fundamental and second harmonic respectively. These amplitudes are assumed to have an order of magnitude $\varepsilon^{l_{0}}$. Furthermore, $\xi, \eta, \zeta, \tau$ have an order of magnitude $\varepsilon$, and $\xi_{2}$ an order $\varepsilon^{2}$. Therefore the left-hand-side of equation (3.11) has the order $\varepsilon^{2+l_{0}}$, and its right-hand-side has the order $\varepsilon^{2 l_{0}}$. We must thus have $2+l_{0}=$ $2 l_{0}$, and $l_{0}=2$.

This differs from the case of the cubic NLS equation, which is

$$
i \partial_{\xi_{2}} f+D_{\vec{\xi}}^{2} f+A f|f|^{2}=0
$$

with analogous notations, $f$ being the modulated amplitude. There we would have $l_{0}+2=$ $3 l_{0}$, thus $l_{0}=1$. This has a very important physical meaning: the present model requires input energy pulses from a smaller range than the NLS solitons obtained either in cubic media by the Kerr effect or in quadratic media by cascading, far from phase-matching [8]. This is due to the fact that the interaction is resonant, or very close to resonance. Note that the multiscale analysis justifies this difference between the required power inputs without making any special assumption about the values of the nonlinear susceptibilities.

\section{Solving the perturbative scheme}

\subsection{A technical point}

The order by order resolution of the perturbative scheme can now be performed. The detail of this derivation is given in the appendix. The vector amplitudes whose evolution 
is under investigation are polarized so that

$$
\vec{E}_{2}^{1}=\left(\begin{array}{c}
0 \\
\varphi \\
0
\end{array}\right), \quad \vec{E}_{2}^{2}=\left(\begin{array}{c}
0 \\
0 \\
\psi
\end{array}\right) .
$$

The transport equation giving the group velocity is expected to appear at order $\varepsilon^{l_{0}+1}=\varepsilon^{3}$. We find that the amplitude $\varphi$ of the fundamental satisfies the partial differential equation

$$
\partial_{\xi} \varphi+\frac{1}{v} \partial_{\tau} \varphi=i \delta_{1} \varphi
$$

The constants $\delta_{1}$ and $v$ are specified below (equation (4.8)). The solution of equation (4.2) is

$$
\varphi\left(\xi, \xi_{1}, \xi_{2}, \tau, \eta, \zeta\right)=\varphi_{0}\left(\xi_{1}, \xi_{2}, \tau-\frac{\xi}{v}, \eta, \zeta\right) e^{i \delta_{1} \xi}
$$

It describes both a propagation at speed $v$ and a linear phase modulation measured by the coefficient $\delta_{1}$.

The expressions of the constants $v$ and $\delta_{1}$ are given by solving the multiscale expansion. They can be interpreted, and written in a convenient way, by using some power series expansion of the wave numbers. We introduce the following notations:

$$
\begin{gathered}
\tilde{n}_{s}^{2}=n^{2}+\sqrt{\varepsilon} \Delta n_{s}^{2}+\varepsilon \hat{\chi}_{1, s}^{(1)}+\varepsilon^{\frac{3}{2}} \hat{\chi}_{\frac{3}{2}, s}^{(1)}+\cdots \quad \text { for } s=o \text { or } e, \\
\tilde{k}_{o}=\tilde{k}_{o}(\omega)=\frac{\omega \tilde{n}_{o}(\omega)}{c}=k_{0, o}+\sqrt{\varepsilon} k_{\frac{1}{2}, o}+\varepsilon k_{1, o}+\cdots, \\
\tilde{k}_{e}=\tilde{k}_{e}(2 \omega)=\frac{2 \omega \tilde{n}_{e}(2 \omega)}{c}=k_{0, e}+\sqrt{\varepsilon} k_{\frac{1}{2}, e}+\varepsilon k_{1, e}+\cdots .
\end{gathered}
$$

For the derivatives of these quantities, the notations used are

$$
k_{p, o}^{\prime}=\frac{d}{d \omega} k_{p, o}(\omega) \quad \text { and } \quad k_{p, e}^{\prime}=\frac{d}{d(2 \omega)} k_{p, e}(2 \omega) .
$$

Then the values of the coefficients are found,

$$
\delta_{1}=k_{1, o}-\frac{k_{0, o} \gamma^{2}}{2} \quad \text { and } \quad \frac{1}{v}=k_{0, o}^{\prime} .
$$

Thus the phase factor $e^{i \delta_{1} \xi}$ accounts for the corrections to the $x$-component of the wave vector $\vec{k}$ at order $\varepsilon$, in norm (term $\left.k_{1, o}\right)$ and in direction $\left(2^{\text {nd }}\right.$ term). Similar corrections appear at following order $\varepsilon^{3+\frac{1}{2}}$, it is the factor $e^{i \rho_{1} \xi_{1}}$ in formula (A.9), and for the second harmonic: the factors $e^{i \rho_{1} \xi_{1}}$ and $e^{i \rho_{2} \xi_{1}}$ in formulas 4.13a and 4.13b). These corrections are a characteristic feature of the kind of "mixed" expansion used here.

Note that it is decisive, for going further in the perturbative expansion, that equation (4.2) has its particular form. The multiscale expansion frame considered describes indeed the long-distance evolution of some state, that is a steady state in the first approximation. Usually, the order zero is a plane wave, invariant in amplitude, thus order one can be considered. The order one presents Galilean invariance, thus order two can be considered. Higher order make sense mainly if order two describes a soliton, which 
is another kind of invariance. Therefore, the arising of a $\xi_{1}$-dependency should a priori forbid to go to the next order, except if this dependency presents some invariance. As an example, an exponentially decreasing $\xi_{1}$-dependency, describing absorption, would reduce all following orders to small linear corrections to this decreasing, that would give account for the main physical behavior. There is, however, some invariance here, because the $\xi_{1}$-dependent factors are unitary phase factors, and we can pursue the expansion.

\subsection{The phase-matching condition}

The first order of the dispersion relation, found at first nonzero order $\varepsilon^{2}$, is

$$
k=\frac{n(\omega) \omega}{c}=\frac{n(2 \omega) \omega}{c} .
$$

Because $k$ is unique, the first order of the phase-matching condition is obtained as

$$
n(2 \omega)=n(\omega) .
$$

The common value of $n(\omega)$ and $n(2 \omega)$ is written $n$ below.

At order $\varepsilon^{2+\frac{1}{2}}$, the anisotropy appears through a condition involving $\alpha k$, the term of order $\sqrt{\varepsilon}$ in the expansion of $k$. We choose, as written above, the fundamental and the second harmonic as ordinary and extraordinary waves respectively. $\alpha$ takes the value

$$
\alpha=\frac{\Delta n_{o}^{2}(\omega)}{2 n^{2}}=\frac{\Delta n_{e}^{2}(2 \omega)}{2 n^{2}} .
$$

Because $\alpha$ is unique, the second order of the phase-matching condition is got at this point. It is

$$
\Delta n_{o}^{2}(\omega)=\Delta n_{e}^{2}(2 \omega) .
$$

Thus the two first orders of the phase-matching condition are a direct consequence of the ansatz, precisely of the unicity of the wave vector in the phase $\phi$ defined by (3.8). A more complicated ansatz involving two fundamental phases could be envisaged. However, it would not be able to give account for the wave interaction. It is indeed proved below that the following terms in the expansion of the linear susceptibility $\hat{\chi}^{(1)}$ must also coincide (see equation (4.16)). The same kind of considerations would prove the same result for first order, if it was not set a priori in the ansatz.

The following order of the phase-matching condition appears at order $\varepsilon^{4}$ when writing down the nonlinear evolution equation for the complex amplitudes $\varphi$ and $\psi$ of the fundamental and second harmonic respectively. They are equations (3.11), while the amplitudes $\varphi$ and $\psi$ satisfy

$$
\begin{aligned}
& \varphi=\varphi_{1}\left(\xi_{2}, \tau-\frac{\xi}{v}-\frac{\xi_{1}}{v_{1}}, \eta, \zeta-\gamma \xi_{1}\right) e^{i\left(\delta_{1} \xi+\rho_{1} \xi_{1}\right)} \\
& \psi=\psi_{1}\left(\xi_{2}, \tau-\frac{\xi}{v}-\frac{\xi_{1}}{v_{1}}, \eta, \zeta-\gamma \xi_{1}\right) e^{i\left(\delta_{2} \xi+\rho_{2} \xi_{1}\right)} .
\end{aligned}
$$

Equations (3.11) are expressed in a coordinate frame that moves with the wave, at a speed equal to the group velocity of the wave up to order $\sqrt{\varepsilon}$. The coordinates are defined by

$$
\tilde{\tau}=\tau-\frac{\xi}{v}-\frac{\xi_{1}}{v_{1}}, \quad \tilde{\eta}=\eta, \quad \tilde{\zeta}=\zeta-\gamma \xi_{1}, \quad \tilde{\xi}_{2}=\xi_{2} .
$$


In this frame, there is no dependency of the amplitudes relative to the variables $\xi$ and $\xi_{1}$. On the other hand, phase factors depending on these variables appear. These factors are

$$
\exp i\left[\left(\delta_{2}-2 \delta_{1}\right) \xi+\left(\rho_{2}-2 \rho_{1}\right) \xi_{1}\right]
$$

in the evolution equation for $\varphi$, and the inverse in the evolution equation for $\psi$. These two equations yield equations (4.21a) and 4.21b respectively, after cancellation of the factors mentioned. This yields two approximate phase-matching conditions, in addition to (4.11): $\delta_{2}=2 \delta_{1}$ and $\rho_{2}=2 \rho_{1}$. The first condition can be written as $k_{1, e}=2 k_{1, o}$, or as

$$
\chi_{e}^{(1)}(2 \omega)=\chi_{o}^{(1)}(\omega)
$$

\subsection{The phase-matching angle}

Conditions (4.10), 4.12) and (4.16) do not involve a phase-matching angle. They state that the approximate phase-matching must be realized "spontaneously" by the material up to order $\varepsilon$. The last of the phase-matching conditions obtained in the previous subsection is

$$
\rho_{2}=2 \rho_{1} \text {. }
$$

It can be satisfied by some particular choice of the angle $\sqrt{\varepsilon} \gamma$ according to

$$
\gamma^{2}=\frac{n c}{\omega} \frac{k_{\frac{3}{2}, e}(2 \omega)-2 k_{\frac{3}{2}, o}(\omega)}{\Delta n_{o}^{2}(\omega)-\Delta n_{o}^{2}(2 \omega)} .
$$

Formula (4.18) coincides with the usual phase-matching condition $(2.5)$, taking into account the power series expansion of $\chi^{(2)}$ and the "spontaneous" approximate phasematching, up to order $\varepsilon$. A deviation from the exact phase-matching of order $\varepsilon^{2}$ is possible. It is described by the terms $B_{1} \varphi$ in equation (4.21a) and $B_{2} \psi$ in equation (4.21b). The present multiscale expansion proves that the model is by no means valid for a larger deviation. In such a case, only a model involving non-resonant interaction can describe the physical phenomenon. Completely integrable models such as the nonlinear Schrödinger equation (NLS) or the Davey Stewartson system (DS I and II) can be derived in this frame, but involve much higher intensities: of order $\varepsilon$ instead of $\varepsilon^{2}$ in the present case [11, 12].

\subsection{The group velocity matching}

The phase-matching is a phase velocity matching, but for short pulses a group velocity matching is also necessary. Without it, the two pulses cross each other without having time enough to interact. Mathematically, the group velocity matching condition appears through the requirement that the variables $\left(\tilde{\tau}, \tilde{\eta}, \tilde{\zeta}, \tilde{\xi}_{2}\right)$ are uniquely defined by equation (4.14). In other words, the frame moving with the pulses, in which equation (3.11) describes the evolution of the waves, must be defined in a unique way, thus must be the same for both waves.

At order $\varepsilon^{3}$, the first order term of the group velocity is found. It is $1 / v=k_{0, o}^{\prime}$ for the fundamental and $1 / v=k_{0, e}^{\prime}$ for the second harmonic. According to the above statements, the velocity $v$ is the same for both waves. If these velocities differ, it is found at next

order that no interaction between the waves is possible. From the formal point of view, 
the $\xi_{1}$ and $\xi_{2}$-dependencies of $\varphi$ and $\psi$ would be linear if both group velocities have not the same value. For this reason, we must have $k_{0, e}^{\prime}=k_{0, o}^{\prime}$ : this means that the group velocities coincide at order zero. Using the zero order phase-matching condition (4.10), this condition can be written in terms of the index $n$ as

$$
n^{\prime}(\omega)=2 n^{\prime}(2 \omega)
$$

The first correction $1 / v_{1}$ to the inverse of the group velocity is obtained at following

order $\varepsilon^{3+\frac{1}{2}}$. As at previous order, this correction is $1 / v_{1}=k_{\frac{1}{2}, o}^{\prime}$ for the fundamental and $1 / v_{1}=k_{\frac{1}{2}, e}^{\prime}$ for the second harmonic. Therefore $k_{\frac{1}{2}, o}^{\prime}=k_{\frac{1}{2}, e}^{\prime}$ or, in terms of the indices,

$$
\left(\Delta n_{o}^{2}\right)^{\prime}(\omega)=2\left(\Delta n_{e}^{2}\right)^{\prime}(2 \omega)
$$

An explicit walk-off appears in the final equations (4.21a)- $4.21 \mathrm{~b}$ ) through the terms involving a first order $\tau$-derivative. It is measured by the difference between $k_{1, o}^{\prime}$ and $k_{1, e}^{\prime}$. As the deviation from phase-matching, the walk-off has a fixed maximal order of magnitude, that of $\varepsilon$. If this group velocity matching is not satisfied, the model yielded by equations (4.21a)-(4.21b) is theoretically no more valid. This means physically that for a pulse size, a power input, and a propagation distance in accordance with the scaling, the two waves do not coexist at the same place during a long enough time, and cannot interact.

\subsection{The asymptotic model}

As mentioned above, the evolution equations of the form (3.11) for the complex amplitudes $\varphi$ and $\psi$ of the fundamental and second harmonic respectively are obtained at order $\varepsilon^{4}$. They reduce to

$$
\begin{gathered}
{\left[2 i k \partial_{\xi_{2}}-B_{1}+2 i k\left(k_{1, o}^{\prime}+\frac{\gamma^{2}}{2 v}\right) \partial_{\tau}\right.} \\
\left.-k k^{\prime \prime} \partial_{\tau}^{2}+\partial_{\eta}^{2}+\partial_{\zeta}^{2}-2 i k \alpha \gamma \partial_{\zeta}\right] \varphi=A_{1} \psi \varphi^{*} \\
{\left[4 i k \partial_{\xi_{2}}-B_{2}+4 i k\left(k_{1, e}^{\prime}+\frac{\gamma^{2}}{2 v}\right) \partial_{\tau}\right.} \\
\left.-\frac{2 i k \gamma}{n^{2}}\left(2 \Delta n_{o}^{2}+\Delta n_{e}^{2}\right) \partial_{\zeta}-2 k k_{0, e}^{\prime \prime} \partial_{\tau}^{2}+\partial_{\eta}^{2}+\partial_{\zeta}^{2}\right] \psi=A_{2} \varphi^{2}
\end{gathered}
$$

(The notations $\Delta n_{s}^{2}, k_{j, s}(s=o, e)$ are defined by equations (4.4) to (4.6)). The coefficients, $2 i k$ or $4 i k$ of $\partial_{\xi_{2}}$, and $-k k^{\prime \prime}\left(=-k_{0, o} k_{0, o}^{\prime \prime}\right)$ or $-2 k k_{0, e}^{\prime \prime}$ of $\partial_{\tau}^{2}$, are the usual coefficients in the NLS models in nonlinear optics. A transverse velocity term, proportional to $\partial_{\zeta} \varphi$, and a longitudinal one, proportional to $\partial_{\tau} \varphi$, appear (the coefficient $\alpha$ is given by equation (4.11)). $\quad k_{1, o}^{\prime}$ and $k_{1, e}^{\prime}$ are the corrections to the inverse of the group velocities at order $\varepsilon$.

The coefficients $B_{1}$ and $B_{2}$ are some corrections to the phase vector at this order. They 
have the expressions

$$
\begin{gathered}
B_{1}=-2 k k_{2, o}+\frac{\omega^{2}}{2 c^{2}}\left[\frac{3\left(\Delta n_{o}^{2}\right)^{2}(\omega)}{4 n^{4}}-\hat{\chi}_{1, o}^{(1)}(\omega)\right] \gamma^{2}+\frac{k^{2}}{4} \gamma^{4} \\
B_{2}=-4 k k_{2, e}+4 k^{2}\left[\frac{1}{2 n^{2}}\left(\hat{\chi}_{1, e}^{(1)}-2 \hat{\chi}_{1, o}^{(1)}\right)\right. \\
\left.-\frac{1}{8 n^{4}}\left(\left(\Delta n_{e}^{2}\right)^{2}+4 \Delta n_{o}^{2} \Delta n_{e}^{2}-8\left(\Delta n_{o}^{2}\right)^{2}\right)\right] \gamma^{2}+k^{2} \gamma^{4} .
\end{gathered}
$$

The nonlinear interaction constant $A_{1}$ and $A_{2}$ are defined in the general case by equations (A.24)-(A.30) (in appendix). Using the symmetry properties of the $\chi^{(2)}$-tensor, including the so-called complete symmetry property, we have $A_{2}=2 A_{1}$, at least for the $\overline{4} 2 \mathrm{~m}$ and $3 \mathrm{~m}$ symmetry classes, for which the common value of these coefficients is given by equations (A.27) and (A.29).

Equations (4.21a) and (4.21b) yield the sought model. They are analogous to the equations given by Menyuk in [14], but here in a complete $(3+1)$-dimensional version. Kanashov and Rubenchik shown [9] that no collapse occurs in this model, and that a soliton solution exists in the sense of a stable localized pulse. System (4.21a)-4.21b is written in a coordinate frame that moves with the wave at a speed equal to its group velocity up to order $\sqrt{\varepsilon}$. It is seen that the group velocity of both fundamental and second harmonic must coincide up to this order. Walk-off terms of order $\varepsilon$ appear in equations (4.21a) and $4.21 \mathrm{~b}$ ). These are the terms involving first order $\tau$ - and $\zeta$-derivatives. In an analogous way, it is seen that the approximate phase-matching must be realized "spontaneously" by the material up to order $\varepsilon$, and once more at order $\varepsilon^{3 / 2}$ by some particular choice of the angle $\sqrt{\varepsilon} \gamma$, according to equation (4.18).

Consider now the expansion (3.10) of the susceptibility tensor, that also has the expression (2.3). Because we must have an isotropic zero order term, $\sqrt{\varepsilon}$ is the order of magnitude of the anisotropy. The interaction must be phase-matched "spontaneously" up to order $\varepsilon$, thus the order of magnitude of the difference $\left(n_{o}(\omega)-n_{e}(2 \omega)\right)$ must be $\varepsilon^{3 / 2}$. Last, the group velocity of both waves must be identical up to order $\sqrt{\varepsilon}$. Thus their difference must have an order of magnitude $\varepsilon$. Finally, the matching conditions are

$$
\begin{gathered}
n_{o}^{2}(\omega)-n_{e}^{2}(\omega) \in O(\sqrt{\varepsilon}), \\
\frac{d n_{o}^{2}(\omega)}{d \omega}-\frac{d n_{e}^{2}(2 \omega)}{d \omega} \in O(\varepsilon), \\
n_{o}^{2}(\omega)-n_{e}^{2}(2 \omega) \in O\left(\varepsilon^{3 / 2}\right) .
\end{gathered}
$$

$\omega_{0}$ is the fundamental frequency and $\varepsilon$ the perturbation parameter.

\section{Conclusion}

A long-distance propagation model (equations (4.21a)- 4.21b ) for two interacting waves with different velocities has been derived through a rigorous multiscale analysis. It uses a new type of expansion that mixes a multiscale expansion, and an expansion in a power series of some physical quantity, that here represents mainly the anisotropy. This expansion involves 5 longitudinal space scales, instead of 3 in the usual NLS-type models. The 
supplementary scale is related to approximate conditions satisfied by the linear susceptibilities. The expansion considered will be useful for the mathematical justification of asymptotic models of the above mentioned type. This rigorous derivation has allowed the determination of the validity conditions of the model.

From a more concrete point of view, the model equations $4.21 \mathrm{a}-4.21 \mathrm{~b}$ ) describe the evolution of the modulation of two short localized pulses, fundamental and second harmonic, propagating together in a bulk uniaxial crystal with non-vanishing second order susceptibility $\chi^{(2)}$, and interacting. Much theoretical work has already been done about this system. It has been proved that no collapse occurs [9, 1], analytical solutions have been found in $(1+1)$ dimensions [14], and numerical simulations have shown the existence of stable solutions [16]. We have determined the physical conditions under which the model is valid: both phase and group velocity of the waves must be close enough together. Group velocities are matched when anisotropy is small. Thus the phase-matching angle must be small. Therefore, the phase-matching must be realized by the intrinsic properties of the material and for any propagation direction with some precision. Hence the model cannot be valid in any material and at any frequency. X-Mozilla-Status: 0000

\section{Appendix. Details of the derivation}

\section{A.1 Dispersion relation}

In this appendix, we give the rigorous derivation of system 4.21a $-(4.21 \mathrm{~b})$, using the perturbative scheme described in Section 2. The convolutions involved by expression (2.2) of $\vec{D}$ are treated using the formulas derived in [11]. We solve it order by order: the first nonzero order, $\varepsilon^{2}$, gives that $E_{2}^{p, s}$ is zero for all $s=x, y, z$, and all integer $p$, except for $p= \pm 1, \pm 2$, and $s=y, z$, if the dispersion relation (4.9) is satisfied.

At order $\varepsilon^{2+\frac{1}{2}}$, a first correction to the dispersion relation is obtained through the ex-

pression (4.11) of the coefficient $\alpha$. The polarization vectors $\vec{E}_{2}^{1}$ and $\vec{E}_{2}^{2}$ are also found, they are defined as (4.1). $\varphi$ and $\psi$ are the amplitudes whose evolution is under investigation, and the other $\vec{E}_{2}^{p}$ are zero (except for the symmetric terms $p=-1,-2$ ).

\section{A.2 Order 3 and group velocity}

At order $\varepsilon^{3}$, the group velocity appears. Precisely, we get as a solvability condition for $\varphi$ the equation (4.2), which admits the solution (4.3). In an analogous way, the $\xi$-dependency of the second harmonic is obtained:

$$
\psi=\psi_{0}\left(\xi_{1}, \xi_{2}, \tau-\frac{\xi}{v}, \eta, \zeta\right) e^{i \delta_{2} \xi}
$$

with

$$
\delta_{2}=k_{1, e}-\frac{k_{0, e} \gamma^{2}}{2} \quad \text { and } \quad \frac{1}{v}=k_{0, o}^{\prime} .
$$

Other conditions give

$$
\vec{E}_{2+\frac{1}{2}}^{1}=\left(\begin{array}{c}
0 \\
f \\
0
\end{array}\right), \quad \vec{E}_{2+\frac{1}{2}}^{2}=\left(\begin{array}{c}
-\gamma \psi \\
0 \\
g
\end{array}\right) .
$$


The functions $f$ and $g$ have to be determined.

\section{A.3 Order $\varepsilon^{3+\frac{1}{2}}$}

The following order $\varepsilon^{3+\frac{1}{2}}$ gives, in a similar way, the term of order $\sqrt{\varepsilon}$ in the group velocity. The following equation is then obtained:

$$
\begin{aligned}
-2 i k \partial_{\xi} f & -2 i k \alpha \partial_{\xi} \varphi-2 i k \partial_{\xi_{1}} \varphi=2 k \delta_{1} f+\frac{2 i k}{V} \partial_{\tau} f \\
+ & \frac{\omega^{2}}{c^{2}} \hat{\chi}_{\frac{3}{2}, o}^{(1)} \varphi+\frac{i \omega}{c^{2}}\left(\left(\Delta n_{o}^{2}\right)^{\prime} \omega+2 \Delta n_{o}^{2}\right) \partial_{\tau} \varphi .
\end{aligned}
$$

Equation (A.4) is solved as follows: using the change of variables

$$
\hat{\tau}=\tau-\frac{\xi}{v}, \quad \hat{\xi}=\xi
$$

noting that $\varphi=\varphi_{0}(\hat{\tau}) e^{i \delta_{1} \hat{\xi}}$ (equation $(4.3)$ ), and setting

$$
f=f_{0}(\hat{\xi}, \hat{\tau}) e^{i \delta_{1} \hat{\xi}}
$$

in equation (A.4), it is found that $\partial_{\hat{\xi}} f_{0}$ expresses in terms of $\varphi_{0}$ and of its derivatives. Thus, relative to $\hat{\xi}, \partial_{\hat{\xi}} f$ is some constant $A$, and

$$
f_{0}=A \hat{\xi}+B
$$

$B$ being some constant. Because $f_{0}$ must be bounded as $\hat{\xi}$ tends to infinity, $A$ must be zero. This implies that $f_{0}$ is a function of $\hat{\tau}$ alone, and this yields some partial differential equation to be satisfied by $\varphi_{0}$ (or $\varphi$ ). This equation is obtained from equation (A.4) by simplifying the terms containing $f$. It is

$$
\partial_{\xi_{1}} \varphi_{0}+\gamma \partial_{\zeta} \varphi_{0}+\frac{1}{v_{1}} \partial_{\tau} \varphi_{0}-i \rho_{1} \varphi_{0}=0
$$

The solution of equation (A.8) is

$$
\varphi_{0}=\varphi_{1}\left(\xi_{2}, \tau-\frac{\xi}{v}-\frac{\xi_{1}}{v_{1}}, \eta, \zeta-\gamma \xi_{1}\right) e^{i \rho_{1} \xi_{1}}
$$

$1 / v_{1}$ is the first correction to the inverse of the group velocity

$$
\frac{1}{v_{1}}=k_{\frac{1}{2}, o}^{\prime}
$$

$\rho_{1}$ is the next correction to the wave vector $k$,

$$
\rho_{1}=k_{\frac{3}{2}, o}+\frac{\omega}{4 n c} \Delta n_{o}^{2}(\omega) \gamma^{2} .
$$

The dependency relative to $\left(\zeta-\gamma \xi_{1}\right)$ does not represent a walk-off: it simply gives account for wave propagation in a direction making the small angle $\gamma \sqrt{\varepsilon}$ with the $x$-axis. 
In an analogous way, the $\xi$-evolution of the second harmonic is found,

$$
g=g_{0}\left(\tau-\frac{\xi}{v}\right) e^{i \delta_{2} \xi}
$$

with

$$
\frac{1}{v_{1}}=k_{\frac{1}{2}, e}^{\prime}
$$

and

$$
\rho_{2}=k_{\frac{3}{2}, e}-\frac{\omega \gamma^{2}}{2 n c}\left(\Delta n_{e}^{2}(2 \omega)-2 \Delta n_{o}^{2}(2 \omega)\right) .
$$

Equation (4.13b) is deduced from equation (A.12). As previously, the variables must be the same in expression (A.9) for $\varphi$, as in (4.13b) for $\psi$. In particular, the correction term $1 / v_{1}$ to the inverse of the group velocity must be the same in both cases. Thus

$$
k_{\frac{1}{2}, o}^{\prime}=k_{\frac{1}{2}, e}^{\prime}
$$

Other conditions give

$$
\begin{aligned}
& \vec{E}_{3}^{1}=\left(\begin{array}{c}
\frac{i}{k} \partial_{\eta} \varphi \\
F \\
0
\end{array}\right) \\
& \vec{E}_{3}^{2}=\left(\begin{array}{c}
-\gamma g+\frac{i}{2 k} \partial_{\zeta} \psi+\frac{\gamma}{2 n^{2}}\left[2 \Delta n_{o}^{2}(2 \omega)-\Delta n_{e}^{2}(2 \omega)\right] \psi \\
0 \\
G
\end{array}\right) .
\end{aligned}
$$

$F, G$ are functions to be determined. The expressions of $E_{3+\frac{1}{2}}^{1, x}, E_{3+\frac{1}{2}}^{2, x}$ are also obtained, they are use in the remainder of the computation:

$$
\begin{gathered}
E_{3+\frac{1}{2}}^{1, x}=\frac{i}{k} \partial_{\eta} f-\frac{\Delta n_{o}^{2}(\omega)}{2 n^{2}} \partial_{\eta} \varphi \\
E_{3+\frac{1}{2}}^{2, x}=-\gamma G+\frac{i}{2 k} \partial_{\zeta} g+\frac{\gamma}{2 n^{2}}\left[2 \Delta n_{o}^{2}-\Delta n_{e}^{2}\right] g \\
+\left[\frac{\Delta n_{o}^{2}}{2 n^{4}}\left(\Delta n_{e}^{2}-2 \Delta n_{o}^{2}\right)+\frac{1}{n^{2}} \hat{\chi}_{1, o}^{(1)}-\gamma^{2}\right] \gamma \psi \\
+\frac{i \gamma}{2 k} \partial_{\xi} \psi+\frac{i}{4 k n^{2}}\left(\Delta n_{e}^{2}-2 \Delta n_{o}^{2}\right) \partial_{\zeta} \psi+\frac{i \gamma}{k v} \partial_{\tau} \psi .
\end{gathered}
$$

(All indices in equation (A.19) are taken at $2 \omega$ ).

\section{A.4 The evolution equations}

At order $\varepsilon^{4}$, the evolution equations for $\varphi$ and $\psi$ are obtained as follows: the equation of order $\varepsilon^{4}$ for the $y$-component of the fundamental is a partial differential equation involving $F, f$, and $\varphi$. Using the variables $\hat{\xi}$ and $\hat{\tau}$ defined by equation (A.5), and writing $F=$ $F_{0}(\hat{\xi}, \hat{\tau}) e^{i \delta_{1} \hat{\xi}}$, it is found that $\partial_{\hat{\xi}} F_{0}$ is equal to some expression depending on $\varphi_{0}, f_{0}$, and their derivatives. This expression does not depend on $\hat{\xi}$. Thus, as shown above for $f_{0}, F_{0}$ is a function of $\hat{\tau}$ only, and the terms depending on $F$ cancel in the equation. 
Then considering equation (A.9), $f_{0}$ is

$$
f_{0}=f_{1}\left(\xi_{1}, \xi_{2}, \tau-\frac{\xi_{2}}{v_{1}}, \eta, \zeta-\gamma \xi_{1}\right) e^{i \rho_{1} \xi_{1}}
$$

The notation $\partial_{\hat{\xi}_{1}}$ holds for the partial derivative relative to $\xi_{1}$ in the variables defined by equation (A.20). Transferring (A.9) and (A.20) into the equation, the following result is obtained: $\partial_{\hat{\xi}_{1}} f_{1}$ is equal to some expression that depends on $\varphi_{1}$ only. Thus $\partial_{\hat{\xi}_{1}} f_{1}$ does not depend on $\xi_{1}$. Because $f_{1}$ must be bounded as $\xi_{1}$ tends to infinity, $f_{1}$ is independent of $\xi_{1}$ too. Then $f_{1}$ disappears from the equation, that reduces to the evolution equation (4.21a) for $\varphi$, taking into account the nonlinear term computed in the following subsection.

The equation for the second harmonic is obtained in a similar way. It comes from the equation of the multiscale expansion at order $\varepsilon^{4}$, for the $z$-component of the second harmonic. In the same way as for the functions $F$ and $f$ in the case of the fundamental, it is seen that the functions $G$ and $g$ must have the form

$$
G=G_{0}\left(\tau-\frac{\xi}{v}\right) e^{i \delta_{2} \xi}
$$

and

$$
g=g_{1}\left(\xi_{2}, \tau-\frac{\xi}{v}-\frac{\xi_{1}}{v_{1}}, \eta, \zeta-\gamma \xi_{1}\right) e^{i\left(\delta_{2} \xi+\rho_{2} \xi_{1}\right)} .
$$

Then all terms involving either $G$ or $g$ cancel in the equation, and the evolution equation (4.21b) for $\psi$ is obtained.

\section{A.5 Computation of the nonlinear terms}

The nonlinear term in equation (4.21a) for $\varphi$ is

$$
-\frac{\omega^{2}}{c^{2}} P_{4}^{1, y}
$$

where $P_{4}^{1, y}$ is the $y$-component in the coefficient $\vec{P}_{4}^{1}$ of $\varepsilon^{4} e^{i \phi}$, in the expansion of the nonlinear polarization $\chi^{(2)}: \vec{E} \vec{E}$. It is straightforwardly seen that

$$
\vec{P}_{4}^{1}=2 \hat{\chi}^{(2)}(2 \omega,-\omega): \vec{E}_{2}^{2} \vec{E}_{2}^{-1} .
$$

The value of this nonlinear term depends on the symmetry properties of the $\chi^{(2)}$-tensor. For the $\overline{4} 2 \mathrm{~m}$ class of crystals, to which belongs e.g. KDP, in the crystal axes frame [4], the non-vanishing components of the $\chi^{(2)}$-tensor are

$$
\chi_{x y z}^{(2)}=\chi_{y x z}^{(2)}, \quad \chi_{x z y}^{(2)}=\chi_{y z x}^{(2)}, \quad \chi_{z x y}^{(2)}=\chi_{z y x}^{(2)} .
$$

and all other $\chi^{(2)}$-components are zero. Thus, if the axes of the coordinates frame are the crystal axes, because $\vec{E}_{2}^{2}$ and $\vec{E}_{2}^{1}$ are parallel to the $z$ - and to the $y$-axis respectively, $\vec{P}_{4}^{2, y}$ would be zero. Thus the crystal axes must be rotated for some angle $\beta$ around the optical axis (the $z$-axis). We call $\left(x^{\prime}, y^{\prime}, z^{\prime}\right)$ this rotated crystal axes frame. Relative to it, the nonlinear term becomes

$$
-\frac{\omega^{2}}{c^{2}} \vec{P}_{4}^{1}=A_{1}\left(\begin{array}{l}
0 \\
0 \\
1
\end{array}\right) \psi \varphi^{*},
$$


with

$$
A_{1}=\frac{2 \omega^{2}}{c^{2}} \sin 2 \beta \chi_{x^{\prime} y^{\prime} z^{\prime}}^{(2)}(2 \omega,-\omega) .
$$

For the $3 \mathrm{~m}$ symmetry class, the nonzero components of the $\chi_{(2)}$-tensor are 四]

$$
\begin{gathered}
\chi_{x z x}^{(2)}=\chi_{y z y}^{(2)}, \quad \chi_{x x z}^{(2)}=\chi_{y y z}^{(2)}, \quad \chi_{z x x}^{(2)}=\chi_{z y y}^{(2)}, \quad \chi_{z z z}^{(2)}, \\
\chi_{y y y}^{(2)}=-\chi_{y x x}^{(2)}=-\chi_{x x y}^{(2)}=-\chi_{x y x}^{(2)} .
\end{gathered}
$$

The coefficient $A_{1}$ is independent from the angle $\beta$ introduced for the $\overline{4} 2 \mathrm{~m}$ class, and has the expression

$$
A_{1}=-\frac{2 \omega^{2}}{c^{2}} \hat{\chi}_{z x x}^{(2)}(\omega, \omega) .
$$

Other values for this constant are obtained with other crystal symmetries. They are easily computed, or can be found e.g. in [6].

In equation (4.21b) for $\psi$, the nonlinear interaction constant $A_{2}$ is defined in the general case by

$$
-\frac{4 \omega^{2}}{c^{2}} P_{4}^{2, z}=A_{2} \varphi^{2}
$$

For both the $\overline{4} 2 \mathrm{~m}$ and the $3 \mathrm{~m}$ symmetry classes, the use of the $\chi^{(2)}$-structure (A.25) or (A.28), and the complete symmetry property of the $\chi^{(2)}$-tensor, proves the equality $A_{2}=2 A_{1}$.

This completes the derivation of the model equations (4.21a $-4.21 \mathrm{~b})$.

\section{References}

[1] Bergé L, Mezentsev V K, Rasmussen J J and Wyller J, Formation of Stable Solitons in Quadratic Nonlinear Media, Phys. Rev. A52 (1995), R28.

[2] Born M and Wolf E W, Principles of Optics, Pergamon Press, Oxford, 1964.

[3] Boyd G D, Ashkin A, Dziedzic J M and Kleinman D A, Second-Harmonic Generation of Light with Double Refraction, Phys. Rev. A137, Nr. 4 (1965), 1305-1320.

[4] Boyd R W, Nonlinear Optics, Academic Press inc., San Diego, 1992.

[5] Colin T, Rigorous Derivation of the Nonlinear Schrödinger Equation and Davey-Stewartson Systems from Quadratic Hyperbolic Systems, Asympt. Analysis, to appear.

[6] Dmitriev V G, Gurzadyan G G and Nikogosyan D N, Handbook of Nonlinear Optical Crystals, Springer, Berlin, 1991.

[7] Dodd R K, Eilbeck J C, Gibbon J D and Morris H C, Solitons and Nonlinear Wave Equations, Academic Press, London, 1982.

[8] Kalocsai A G and Haus J W, Nonlinear Schrödinger Equation for Optical Media with Quadratic Nonlinearity, Phys. Rev. A49, Nr. 1 (1994), 574-585.

[9] Kanashov A A and Rubenchik A M, On Diffraction and Dispersion Effect on Three Waves Interaction, Physica D4 (1981), 122-134. 
[10] Leblond H, Electromagnetic Waves in Ferrites: from Linear Absorption to the Nonlinear Schrödinger Equation, J. Phys. A: Math. Gen. 29 (1996), 4623-4639.

[11] Leblond H, Propagation of Optical Localized Pulses in $\chi^{(2)}$ Crystals: a $(3+1)$-Dimensional Model and Its Reduction to the NLS Equation, J. Phys. A: Math. Gen. 31 (1998), 3041-3066.

[12] Leblond H, Bidimensionsal Optical Solitons in a Quadratic Medium, J. Phys. A: Math. Gen. 31 (1998), 5129-5143.

[13] Leblond H, Parametric Light Bullets: a Multiscale Analysis, J. Opt. A: Pure Appl. Opt. 4, Nr. 2 (2002), 160-173.

[14] Menyuk C R, Schiek R and Torner L, Solitary Waves due to $\chi^{(2)}: \chi^{(2)}$ Cascading, J. Opt. Soc. Am. B11, Nr. 12 (1994), 2434-2443.

[15] Nakata I, Shock Waves in a Ferromagnet, J. Phys. Soc. Jpn. 60 (1991), 2179-2183.

[16] Torner L, Mihalache D, Mazilu D, Wright E M, Torruellas W E and Stegeman G I, Stationary Trapping of Light Beams in Bulk Second-Order Nonlinear Media, Optics Communications 121 (1995), 149-155.

[17] Yariv A and Yeh P, Optical Waves in Crystals, Propagation and Control of Laser Radiation, Wiley-Interscience, New York, 1983. 